\title{
The Puzzling Frequencies of CEMP and NEMP Stars
}

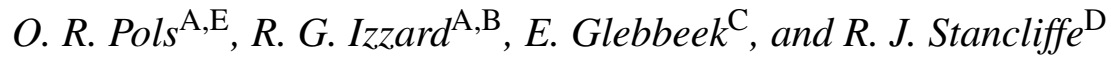 \\ A Sterrekundig Instituut Utrecht, P.O. Box 80000 , NL-3584 TA Utrecht, The Netherlands \\ B Institut d'Astronomie et d'Astrophysique, Université Libre de Bruxelles, CP226, \\ Boulevard du Triomphe, B-1050 Bruxelles, Belgium \\ ${ }^{\mathrm{C}}$ Department of Physics and Astronomy, McMaster University, Hamilton, Ontario, \\ L8S 4M1, Canada \\ D Centre for Stellar and Planetary Astrophysics, Monash University, P.O. Box 28M, \\ Clayton, VIC 3800, Australia \\ E Corresponding author. Email: o.r.pols@uu.nl
}

Received 2008 December 23, accepted 2009 April 9

\begin{abstract}
We present the results of binary population simulations of carbon- and nitrogen-enhanced metalpoor (CEMP and NEMP) stars. We show that the observed paucity of very nitrogen-rich stars puts strong constraints on possible modifications of the initial mass function at low metallicity.
\end{abstract}

Keywords: stars: AGB and post-AGB — stars: abundances — stars: binaries — stars: evolution — stars: mass function

\section{Introduction}

Carbon-enhanced metal-poor (CEMP) stars make up at least $10 \%$ and probably as much as $20-25 \%$ of very metal-poor stars with $[\mathrm{Fe} / \mathrm{H}]<-2$ (Frebel et al. 2006; Lucatello et al. 2006). These stars have $[\mathrm{C} / \mathrm{Fe}]>1.0$, and about $80 \%$ of CEMP stars are also enriched in $s$-process elements (CEMP-s stars; Aoki et al. 2007). A likely formation scenario is pollution by mass transfer from a more massive AGB companion in a binary system, which has since become a white dwarf. Radial velocity monitoring indeed suggests that probably all CEMP-s stars are binaries (Lucatello et al. 2005a).

Within the mass transfer scenario, the large proportion of CEMP-s stars requires the existence of a sufficient number of binary systems with primary components that have undergone AGB nucleosynthesis. In recent studies (Lucatello et al. 2005b; Komiya et al. 2007) it has been argued that a different initial mass function (IMF) is therefore needed at low metallicity, weighted towards intermediate-mass stars. If true, this in turn has important consequences for the chemical evolution of the halo and of other galaxies. However, the model calculations on which these estimates are based still contain many uncertainties regarding the evolution and nucleosynthesis of low-metallicity AGB stars, the efficiency of mass transfer, and the evolution of the surface abundances of the CEMP stars themselves (e.g., see Izzard et al., this volume).

Apart from carbon, substantial enhancements of nitrogen with respect to iron are common among CEMP stars, typically with $[\mathrm{C} / \mathrm{N}]>0$. Detailed AGB nucleosynthesis models of low initial mass $\left(<2.5 \mathrm{M}_{\odot}\right)$ produce carbon, but do not produce nitrogen because it is burned during helium shell flashes. On the other hand, AGB models of higher mass convert the dredged-up carbon into nitrogen by $\mathrm{CN}$ cycling at the bottom of the convective envelope (hot bottom burning, HBB). The surface abundances of these more massive AGB stars approach the $\mathrm{CN}$ equilibrium ratio of $[\mathrm{C} / \mathrm{N}] \approx-2$. Detailed evolution models of AGB stars (Karakas \& Lattanzio 2007) indicate that, while at solar metallicity $\mathrm{HBB}$ sets in at $\gtrsim 5 \mathrm{M}_{\odot}$, significantly lower masses are needed at low metallicity $\left(\gtrsim 2.7 \mathrm{M}_{\odot}\right.$ at $[\mathrm{Fe} / \mathrm{H}]=-2.3)$. One may thus expect a population of socalled nitrogen-enhanced metal-poor (NEMP) stars, with $[\mathrm{N} / \mathrm{Fe}]>0.5$ and $[\mathrm{C} / \mathrm{N}]<-0.5$. Although a few examples of such stars are known, mostly at $[\mathrm{Fe} / \mathrm{H}]<-2.9$, they appear to be very rare (Johnson et al. 2007).

In this contribution we show that the number of NEMP stars sets an additional constraint on possible changes to the IMF at low metallicity. In Sect. 2 we present results of our binary population synthesis simulations, and in Sect. 3 we give our conclusions.

\section{Binary Population Nucleosynthesis Modelling}

We have simulated populations of metal-poor halo stars in binary systems using the rapid synthetic binary nucleosynthesis code of Izzard et al. (2004) and Izzard et al. (2006). The algorithm and the assumptions made in these simulations are briefly summarized by Izzard et al. (this volume). We adopt a metallicity $Z=10^{-4}([\mathrm{Fe} / \mathrm{H}]=-2.3)$ and select stars with ages between 10 and $13.7 \mathrm{Gyr}$ (roughly corresponding to the age of the halo) and $\log g<4.0$. Among this sample we designate as CEMP stars those with $[\mathrm{C} / \mathrm{Fe}]>1.0$ and as NEMP stars those with $[\mathrm{N} / \mathrm{Fe}]>0.5$ and $[\mathrm{C} / \mathrm{N}]<-0.5$, following the definition of Johnson et al. (2007). Note that these definitions partly overlap. 
Table 1. Number fractions of CEMP and NEMP stars among halo stars at $[\mathrm{Fe} / \mathrm{H}]=-2.3$

\begin{tabular}{lccc}
\hline Model & CEMP & NEMP & NEMP/CEMP \\
\hline 1A & $2.30 \%$ & $0.35 \%$ & 0.15 \\
1B & $3.50 \%$ & $0.71 \%$ & 0.20 \\
1C & $3.15 \%$ & $0.62 \%$ & 0.20 \\
1D & $4.81 \%$ & $1.35 \%$ & 0.28 \\
1E & $13.5 \%$ & $26.6 \%$ & 2.0 \\
2A & $9.4 \%$ & $0.34 \%$ & 0.04 \\
2B & $8.5 \%$ & $0.70 \%$ & 0.08 \\
2C & $10.5 \%$ & $0.61 \%$ & 0.06 \\
2D & $12.5 \%$ & $1.33 \%$ & 0.11 \\
2E & $15.8 \%$ & $26.4 \%$ & 1.7 \\
\hline
\end{tabular}

We adopt a binary fraction of $100 \%$; for different assumptions the resulting numbers of CEMP and NEMP stars should simply be scaled with the assumed binary fraction.

We compare our model results with the statistics of the SAGA database of metal-poor stars (Suda et al. 2008). We selected 375 stars from the database in a metallicity range $[\mathrm{Fe} / \mathrm{H}]=-2.3 \pm 0.5$ and $\log g<4.0$. Of these, 296 have a $\mathrm{C}$ abundance measurement and 69 classify as CEMP stars, yielding a CEMP fraction of $18-23 \%$, while $88 \%$ of these classify as CEMP-s (defined as having $[\mathrm{Ba} / \mathrm{Fe}]>0.5)$. Only one star classifies as a NEMP star, giving a very small nominal NEMP fraction of $\sim 0.3 \%$ and a ratio of NEMP to CEMP stars of $\sim 0.015$. If we consider an extended metallicity range $-4<[\mathrm{Fe} / \mathrm{H}]<-2$ in order to improve the number statistics, we find 7 NEMP stars and 78 CEMP stars, giving a NEMP fraction of about $1.5 \%$ and a NEMP to CEMP ratio of 0.09 , which we regard as an upper limit to the NEMP/CEMP ratio at $[\mathrm{Fe} / \mathrm{H}] \approx-2.3$. We note that the ratio of NEMP to CEMP stars is a better constraint on our model predictions, because it is independent of various model uncertainties, most notably the binary fraction.

The SAGA database is by no means a statistically complete sample. Bright giants are clearly overrepresented with respect to fainter giants and turnoff stars, compared to what is expected from the relative lifetimes of these phases. However, neither the observed sample nor our model results show a strong dependence of the CEMP fraction on evolution state (represented e.g. by the effective gravity). Therefore the results we describe below are probably not greatly affected by this selection effect.

In Table 1 we give the number fractions of CEMP stars and NEMP stars resulting from our models, for various assumptions regarding the physical ingredients (models 1 and 2) and the initial distributions of binary parameters (A-E). Model 1 is our default model, where we parameterize third dredge-up (3DUP) according to detailed models by Karakas, Lattanzio \& Pols (2002), yielding C-rich AGB stars for initial masses $>1.2 \mathrm{M}_{\odot}$. In Model 2 we assume much more efficient 3DUP in low-mass AGB stars, applying the modifications described in Izzard et al. (this volume). This results in almost all stars with initial masses $0.85-2.7 \mathrm{M}_{\odot}$ becoming C-rich AGB stars and thus potential CEMP progenitors.

The adopted binary parameter distributions are as follows:

A Default model: the initial primary masses $M_{1}$ are distributed according to the solar neighbourhood IMF as derived by Kroupa, Tout \& Gilmore (1993), the initial separations are drawn from a flat distribution in $\log a$ (with $a$ between 3 and $10^{5} \mathrm{R}_{\odot}$ ) and the initial mass ratios from a flat distribution in $q=M_{2} / M_{1}$.

B Primary masses are distributed as in Model A, but mass ratios and orbital periods are drawn from the distributions derived by Duquennoy \& Mayor (1991) for the local population of $\mathrm{G}$ dwarfs, i.e. a log-normal period distribution with a broad peak at 170 years and a mass-ratio distribution with a broad peak at $M_{2} / M_{1}=0.23$.

C Primary masses are taken from an alternative IMF for the solar neighbourhood by Miller \& Scalo (1979), described by a log-normal distribution with a median mass of $0.1 \mathrm{M}_{\odot}$ and a width $\Delta \log M=0.67$, while separations and mass ratios are distributed as in Model A.

D As Model C, but with a modified log-normal IMF with a median mass of $0.79 \mathrm{M}_{\odot}$ and $\Delta \log M=0.5$, as proposed by Lucatello et al. (2005b).

E As Model $\mathrm{C}$ using a log-normal IMF with a much larger median mass of $10 \mathrm{M}_{\odot}$ and $\Delta \log M=0.33$, as proposed by Komiya et al. (2007).

The models with default binary parameter distributions (1A and $2 \mathrm{~A}$ ) are among those presented by Izzard et al. (this volume). As discussed in that paper, our default model (1A) fails to account for the large observed CEMP frequency, while Model 2A leads to an increase of the CEMP fraction to almost $10 \%$, much closer to but still short of the observed value. In both models the small overall NEMP fraction is consistent with the observations, but only Model 2A, with enhanced dredge-up, can reproduce the observed small NEMP/CEMP ratio.

Distributions $B$ and $C$ give some insight into the dependence of our results on uncertainties in the local (solarneighbourhood) binary parameter distributions. Model 1B leads to an increase by a factor of 1.5 in the number of CEMP stars and a factor of 2 in the number of NEMP stars compared to Model 1A, as the peak in the Duquennoy \& Mayor (1991) period distribution coincides with the period range in which mass transfer is effective. On the other hand, in Model 2B the number of CEMP stars is somewhat smaller than in Model 2A because most CEMP stars now come from binaries with primary masses $0.85-1.2 \mathrm{M}_{\odot}$ and $q>0.7$, which is disfavoured by the mass-ratio distribution. Adoption of the Miller \& Scalo (1979) IMF (Models 1C and 2C) also gives somewhat higher CEMP and NEMP fractions than the Kroupa et al. (1993) IMF, but the effect is modest.

The results for distributions $\mathrm{D}$ and $\mathrm{E}$ show the effect of a modified IMF. Model 1D assumes the IMF that Lucatello et al. (2005b) suggest is required to reproduce the large 
CEMP fraction. The number of CEMP stars increases by a factor 2, but still falls short of the observed value. The discrepancy between our and Lucatello's results arises mainly because in our default model the initial primary mass and period range contributing to CEMP stars are smaller than they assumed. With enhanced third dredge-up (Model 2D) the increase is only a modest 30\%. Models 1D and 2D also show an increased NEMP fraction, by a factor 4 over Model A, which is the result of a larger weight of intermediate-mass stars in this IMF. The NEMP/CEMP ratio is too large to be compatible with observations. This effect is much more extreme when we assume the IMF suggested by Komiya et al. (2007) which has a median mass of $10 \mathrm{M}_{\odot}$. Although this gives rise to a substantial CEMP fraction, close to being compatible with observations without the need to assume enhanced dredge-up (Model 1E), the CEMP stars are outnumbered by NEMP stars by a factor of two. This is clearly incompatible with the observed limits on the number fraction of NEMP stars.

\section{Conclusions}

Our binary population synthesis models show that the paucity of NEMP stars among metal-poor halo stars is incompatible with a strongly modified IMF at low metallicity, heavily weighted towards intermediate-mass stars. The observed limit on the ratio of NEMP to CEMP stars constrains allowed changes in the IMF to a median mass of at most $\sim 0.8 \mathrm{M}_{\odot}$, the effect of which on the frequency of CEMP stars is modest. A more promising explanation for both the ubiquity of CEMP-s stars and the near-absence of NEMP stars is that low-mass, low-metallicity AGB stars undergo much more efficient dredge-up of carbon than shown by detailed evolution models available to date (also see Izzard et al., this volume), perhaps as a result of proton ingestion during the first thermal pulse (Cristallo et al., this volume).

\section{Acknowledgments}

We thank Falk Herwig, Maria Lugaro, Amanda Karakas and Selma de Mink for enlightening discussions and useful feedback on the results presented here. R.G.I.'s work in Utrecht is supported by the NWO under grant 614.000.424. R.J.S. is funded by the Australian Research Council's Discovery Projects scheme under grant DP0879472.

\section{References}

Aoki, W., Beers, T. C., Christlieb, N., Norris, J. E., Ryan, S. G. \& Tsangarides, S., 2007, ApJ, 655, 492

Duquennoy, A. \& Mayor, M., 1991, A\&A, 248, 485

Frebel, A., Christlieb, N., Norris, J. E., Aoki, W. \& Asplund, M., 2006, ApJ, 652, 1585

Izzard, R. G., Tout, C. A., Karakas, A. I. \& Pols, O. R., 2004, MNRAS, 350, 407

Izzard, R. G., Dray, L. M., Karakas, A. I., Lugaro, M. \& Tout, C. A., 2006, A\&A, 460, 565

Johnson, J. A., Herwig, F., Beers, T. C. \& Christlieb, N., 2007, ApJ, 658,1203

Karakas, A. I., Lattanzio, J. C. \& Pols, O. R., 2002, PASA, 19, 515

Karakas, A. I. \& Lattanzio, J. C., 2007, PASA, 24, 103

Komiya, Y., Suda, T., Minaguchi, H., Shigeyama, T., Aoki, W. \& Fujimoto, M. Y., 2007, ApJ, 658, 367

Kroupa, P., Tout, C. A. \& Gilmore, G., 1993, MNRAS, 262, 545

Lucatello, S., Tsangarides, S., Beers, T. C., Carretta, E., Gratton, R. G. \& Ryan, S. G., 2005a, ApJ, 625, 825

Lucatello, S., Gratton, R. G., Beers, T. C. \& Carretta, E., 2005b, ApJ, 625,833

Lucatello, S., Beers, T. C., Christlieb, N., Barklem, P. S., Rossi, S., Marsteller, B., Sivarani, T. \& Lee, Y. S., 2006, ApJ, 652, L37

Miller, G. E. \& Scalo, J. M., 1979, ApJS, 41, 513

Suda, T. et al., 2008, PASJ, 60, 1159 